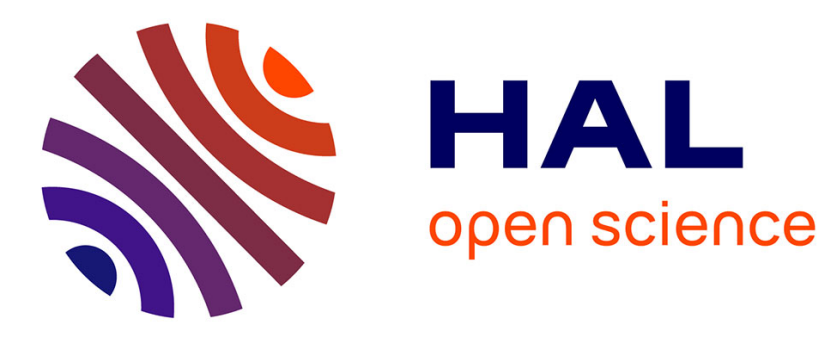

\title{
Performance and design of robust platoons under different communication technologies
}

\author{
Tiago R Gonçalves, Vineeth Satheeskumar Varma Satheeskumar Varma, \\ Salah Eddine Elayoubi
}

\section{To cite this version:}

Tiago R Gonçalves, Vineeth Satheeskumar Varma Satheeskumar Varma, Salah Eddine Elayoubi. Performance and design of robust platoons under different communication technologies. IEEE VTC2021Spring, Apr 2021, Helsinki (virtual), Finland. hal-03199930

\section{HAL Id: hal-03199930 \\ https://hal.science/hal-03199930}

Submitted on 16 Apr 2021

HAL is a multi-disciplinary open access archive for the deposit and dissemination of scientific research documents, whether they are published or not. The documents may come from teaching and research institutions in France or abroad, or from public or private research centers.
L'archive ouverte pluridisciplinaire HAL, est destinée au dépôt et à la diffusion de documents scientifiques de niveau recherche, publiés ou non, émanant des établissements d'enseignement et de recherche français ou étrangers, des laboratoires publics ou privés. 


\section{Performance and design of robust platoons under different communication technologies}

\author{
$1^{\text {st }}$ Tiago R. Gonçalves \\ Laboratory of Signal and Systems \\ Université Paris-Saclay \\ Gif-sur-Yvette, France \\ tiago.rochagoncalves@centralesupelec.fr
}

\author{
$2^{\text {nd }}$ Vineeth S. Varma \\ Control Identification Diagnosis \\ Université de Lorraine \\ Nancy, France \\ vineeth.satheeskumar-varma@univ-lorraine.fr
}

\author{
$3^{\text {rd }}$ Salah E. Elayoubi \\ Laboratory of Signal and Systems \\ Université Paris-Saclay \\ Gif-sur-Yvette, France \\ salaheddine.elayoubi@centralesupelec.fr
}

\begin{abstract}
This article studies cooperative vehicle platooning under different communications approaches and introduces a new dynamic control scheme based on the Predicted Cooperative Adaptive Cruise Control (PCACC). We start by a performance comparison of different communication technologies including VLC (Visible Light Communication) and V2V (Vehicle-toVehicle), with or without relaying via RSU (Road Side Unit), and assess their suitability to fully cooperative and semi-autonomous control schemes. Different from the state of the art, our main design goal is the robustness in terms of an extremely low probability of emergency braking under varying communication conditions and bursts of packet losses. In the light of the performance results, we propose an adaptive control mechanism that tunes the control gains as a function of the observed radio link quality and demonstrate, via extensive simulations, a significantly better performance compared to static control strategies.
\end{abstract}

Index Terms-Vehicle platoons, cooperative adaptive cruise control (CACC), wireless communication.

\section{INTRODUCTION}

In recent years, the field of Intelligent Transportation Systems (ITS) has emerged as a research trending topic to enhance and address new traffic-system challenges [1]. Our particular interest in this paper is the platooning scenario which takes advantages of the particular distribution of a convoy in order to increase road capacity and to decrease fuel consumption, which is achieved by reducing the inter-vehicular distance in order to reduce the air resistance of the platoon's members. The exchange of information is crucial to the deployment of platoons as it allows taking control actions based on the most up-to-date information about the road and traffic status.

\section{A. Paper objectives and contributions}

The first objective of this paper is to evaluate different communication methods between vehicles in platoons to transmit data as illustrated in Fig. 1. The first considered technology is the Visible Light Communication (VLC), in which each vehicle in the platoon exchanges digital information through visible light with the preceding vehicle [2]-[3]. The second method is Vehicle-to-Vehicle (V2V) which enables the communication between vehicles as long as they are in a certain range. The third method extends direct V2V communication by relaying through the network, and we label it RSUr (Road Side Unit relaying). This model involves relaying the information through a base station known as RSU in the ITS terminology or as eNodeB/gNodeB in the 3GPP terminology. In this scenario, we assume that all vehicles in the platoon are in the range of a base station that provides control information to the vehicles to complement the $\mathrm{V} 2 \mathrm{~V}$ communication. Note that there are two main $\mathrm{V} 2 \mathrm{~V}$ communication technologies for ITS, provided by IEEE $802.11 \mathrm{p}$ and 3GPP C-V2X. However, it is not in the scope of this paper discuss the technologies aspects, releases and differences between them. In addition to this performance comparison, the main novelty of this paper is the introduction of a dynamic control mechanism where some of the parameters of the PCACC controller are adapted based on the quality of the V2V communication channel. Packet errors are indeed inherent to all communication channels and may alter the quality of the cooperative controller. We propose a new dynamic approach that adopts the control parameters based on the observed link quality. This new controller comes with different flavours: a centralized scheme based on an offline optimization of the control parameters and a distributed controller where each vehicle adapts its own control parameters based on each communication link.

\section{B. Positioning with respect to the state of the art}

Several works considered the platooning scenario under different communication approaches. For instance, [4] used the $802.11 \mathrm{p}$ technology to evaluate the communication performance under a Cooperative Adaptive Cruise Control (CACC) controller in platoons. Likewise, [5] compared the performances of IEEE 802.11p and 3GPP C-V2X in terms of the inter-vehicular distance of the platoon. In our work, we go beyond these considerations and present an extensive performance comparison of communication modes for different control schemes, including semi-autonomous and fully cooperative control. Our primary performance metric is safety, in terms of avoiding emergency braking. This translates to robustness constraints, where the inter-vehicle distance in the platoon is set so that emergency braking is avoided in 99,999\% of the cases.

As of the conception of control schemes in the presence of degraded radio link quality, [6] proposed a control strategy for graceful degradation based on estimating the preceding vehicle's acceleration, while [7] evaluated the impact of packet 


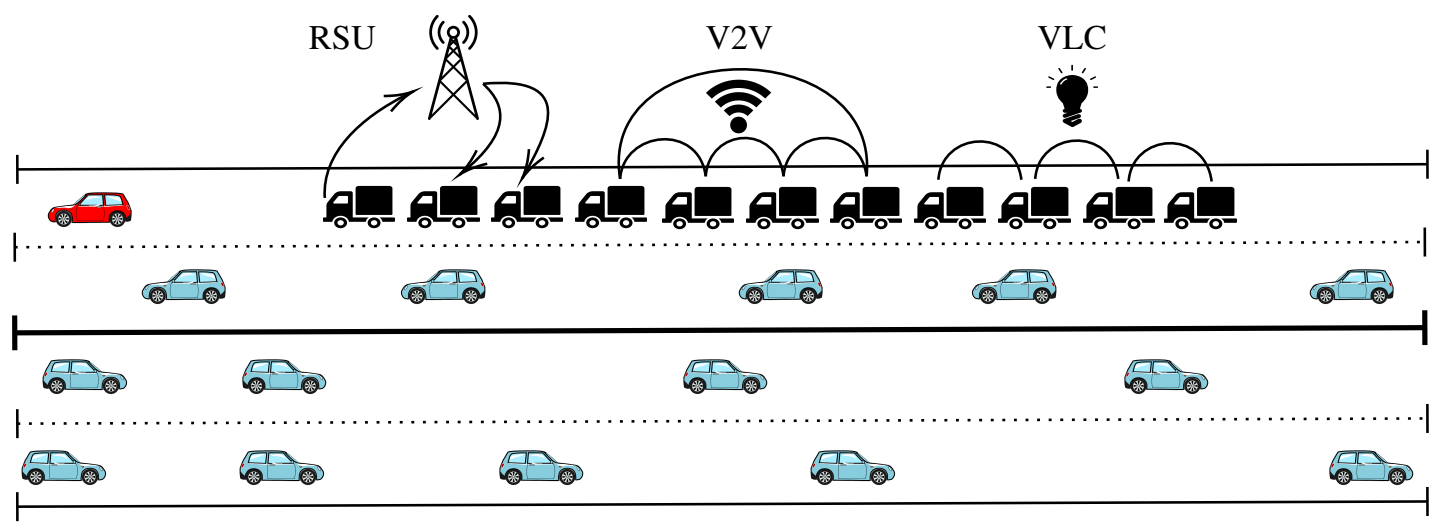

Fig. 1. Traffic scenario and illustration of the different communication approaches: VLC, V2V and RSU relaying.

losses on CACC string stability. [8] suggested different information management algorithms including one with a dynamical control parameter where they simply suggest a lower bound value for it. Our proposed dynamic controller goes far beyond a simple performance assessment under errors or a graceful degradation framework that switches to a completely local controller, as it continuously adapts to the link quality while preserving the robustness. Note that in a previous work [9], we proposed a centralized design for the controller and showed that the radio link quality has a significant impact on the performance, while we present in this paper a more dynamic approach where the controller is dynamically adapted to the communication link status.

\section{Organisation}

The paper is organized as follows. An overview of the control aspects is presented in Section II. Section III introduces the vehicle dynamics, the platoon scenario, the simulation tool adopted and the performance evaluation for different communication methods. Section IV introduces the proposed dynamic control. Finally, we present the conclusions with considerations for future research work.

\section{Control And Communication Platooning SCENARIOS}

This section aims to present vehicular communication technologies and their interaction with the control algorithm's performance. Note that in the control field, the term string stability is consistently used. It implies that any acceleration or braking in the first vehicle is not going to cause an amplification of the error along the tail of the platoon. In other words, as long as the first vehicle is able to avoid a collision all others will be able too. More information about mathematical definitions and conditions to ensure the string stability can be found in [10].

\section{A. Fully Cooperative Control}

One of the most popular platoon controllers is the slidingsurface Cooperative Adaptive Cruise Control (CACC) introduced in [11], where the acceleration of the vehicle is computed based on a weighted sum of factors and its control law is given by

$$
\begin{aligned}
a_{i_{-} d e s} & =C a_{0}+(1-C) a_{i-1}-\omega_{n}^{2}\left(x_{i}-x_{i-1}+D_{d e s}\right) \\
& -\left(2 \xi-C\left(\xi+\sqrt{\xi^{2}-1}\right)\right) \omega_{n}\left(v_{i}-v_{i-1}\right) \\
& -\left(\xi+\sqrt{\xi^{2}-1}\right) \omega_{n} C\left(v_{i}-v_{0}\right)
\end{aligned}
$$

where $D_{\text {des }}$ is the desired inter-vehicular distance that we want to minimize and the index $i$ symbolizes the vehicle index, the first vehicle (the leader) being numbered $0 .\left(x_{i}, v_{i}, a_{i}\right)$ denote the position, velocity, and acceleration of vehicle $i$, respectively. The control parameters to be tuned are $C, \xi$ and $\omega_{n}$. Among the parameters of this controller, an important one is $C$ that corresponds to the weight given to the leader's acceleration in the computation of each vehicle acceleration. $\xi$ is the controller damping ratio and $\omega_{n}$ is the controller bandwidth.

Aiming at improvements, [5] introduced the Predictive CACC (PCACC), while [12] performed a string stability analysis for it. The PCACC controller differs from CACC by the usage of the desired acceleration of the leader and the preceding vehicle instead of the actual value. In other words, the instantaneous acceleration values in (1) are replaced by the desired ones. The PCACC is expected to be superior to the CACC because the actuation lag of the system does not affect directly the control effort which is a big limiting factor for achieving short inter-vehicle distances [5]. For simplicity, we will use the term fully cooperative control when referring to this scheme, as it includes the communication between all the vehicles in the platoon and the leader.

Note that, in all platooning systems, a basic Adaptive Cruise Control (ACC) is adopted by the leader. Its role is to establish a safe distance with the preceding vehicle that is not subject to the platooning controller.

\section{B. Semi-Autonomous Control}

Semi-autonomous control is a particular class of control that relies on the information of the preceding vehicle only, and does not utilize the leader's information. The control law is based on one vehicle look-ahead communication topology, which means that only preceding vehicle's information is required. In this case, its acceleration, velocity, and position are necessary, but without any information from the leader. In 


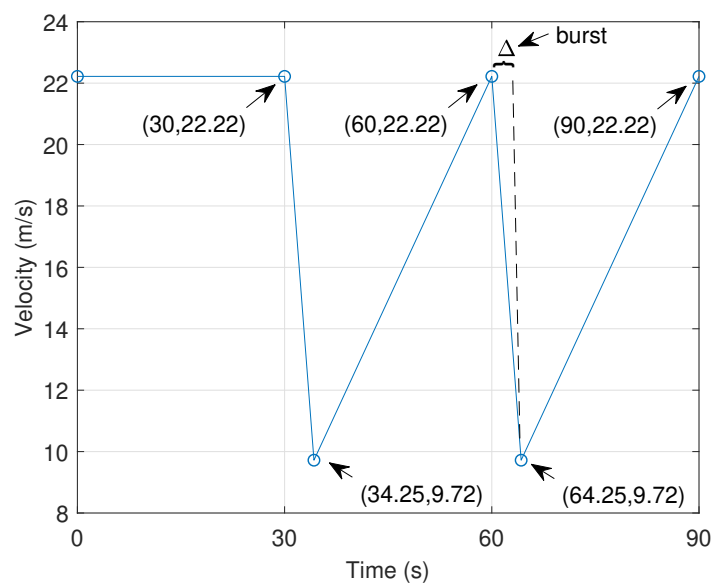

Fig. 2. Illustration of the jammer velocity profile adopted with 2 cycles.

practice, this control strategy can be obtained with a simple change in one of the control parameters of the fully cooperative control, i.e. making the weight of the leader information equal to zero in (1). As proven in [13], this particular control law is string stable when the communication between vehicles is perfect and without delay.

\section{PERFormancE EVAlUATION OF PLATOONING SCHEMES}

Having presented the different platooning schemes, we study in this paper their performances under different communication scenarios, which will give us insights about the optimal control scheme that we will introduce next.

\section{A. Scenarios and environment}

1) Vehicle dynamics: The desired acceleration computed as the output of the CACC controller in (1) is not applied directly because of the actuation lag. Therefore, the lag is modeled as a first-order low pass filter applied to the output of the CACC control:

$$
A_{i}(s)=\frac{1}{\tau s+1} A_{i_{-} d e s}(s)
$$

where $\tau$ is the time constant of the first-order low pass filter $A_{i}$ is the output, which can be interpreted as the actual vehicle acceleration, whereas $A_{i_{-} \text {des }}$ is the vehicle input, which can be seen as the desired acceleration. Note that, $A_{i}(s)$ denotes the Laplace transform of the $a_{i}$ time-domain variable. The idea is to approximate the dynamics of the throttle body and vehicle inertia in order to avoid instantaneous response. In this paper, we assumed a lag of $\tau=0.5 \mathrm{~s}$ as in [10]. The reader can refer the upper level controller in [11] for more details.

2) Platoon scenario and Robustness Criterion: The simulated scenario takes place in a four-lane highway, as illustrated in Fig. 1, with a maximum traffic density of 20 interfering vehicles $/ \mathrm{km}$. The system consists of a platoon of 10 automated vehicles following the leader. The vehicles of all other lanes are not in platoons (blue vehicles) and a jammer (in red) precedes the platoon leader. In the considered scenario, the speed of the jammer follows a preset sequence as in Fig. 2.
TABLE I

COMMUNiCATION AND CONTROLlER PARAMETERS

\begin{tabular}{|c|c|c|c|}
\hline \multicolumn{2}{|c|}{ Communication } & \multicolumn{2}{|l|}{ Controller } \\
\hline Parameter & Value & Parameter & Value \\
\hline Simulation & & PCACC (Followers) & \\
\hline Duration $\left(T_{\text {sim }}\right)$ & $1200 \mathrm{~s}$ & Weight of the leader $(C)$ & Adaptive \\
\hline Jammer profile & Check Fig. 2 & Desired distance $\left(D_{d e s}\right)$ & Adaptive \\
\hline Application & & Damping ratio $(\xi)$ & 2 \\
\hline CAM size & 500 bytes & Bandwidth $\left(\omega_{n}\right)$ & $0.5 \mathrm{~Hz}$ \\
\hline CAM interval & $100 \mathrm{~ms}$ & ACC (Leader) & \\
\hline PHY layer & & Time gap $(h)$ & $1.4 \mathrm{~s}$ \\
\hline Path loss & Winner+B1 LOS & Vehicle \& Highway & \\
\hline Noise power & $-174 \mathrm{dBm} / \mathrm{Hz}$ & Actuator lag $(\tau)$ & $0.5 \mathrm{~s}$ \\
\hline Tx power & $22.5 \mathrm{dBm}$ & Vehicle length $(L)$ & $16.5 \mathrm{~m}$ \\
\hline MCS & QPSK, $\mathrm{R}=1 / 2$ & Max. acceleration & $+2 \mathrm{~m} / \mathrm{s}^{2}$ \\
\hline Channel & Highway NLOS & Min. acceleration & $-3 \mathrm{~m} / \mathrm{s}^{2}$ \\
\hline Frequency $\left(f_{c}\right)$ & $5.9 \mathrm{GHz}$ & Radar interval & $60 \mathrm{~ms}$ \\
\hline Bandwidth (BW) & $10 \mathrm{MHz}$ & Lanes per direction & 2 \\
\hline Process delay & $1 \mathrm{~ms}$ & Lane width & $5 \mathrm{~m}$ \\
\hline & & Traffic density & $20 \mathrm{cars} / \mathrm{km}$ \\
\hline
\end{tabular}

The main reason for this jammer velocity profile is to study the capacity of the platoon system to avoid a collision in risky scenarios such as when the vehicle outside of the convoy suddenly applies the maximum brake capacity. Furthermore, different from the aforementioned papers that perform MonteCarlo simulations (100 or 1000 iterations of normal conditions), we have considered a burst of packet losses. While bursts are rare events, they may occur and alter the safety of the platoon, but are not well reflected in the numerical analysis of most previous works like [4]-[14]. In particular, the robustness considered here is related to the following worstcase event: the jammer brakes at some time and this braking coincides with a burst of packet losses (complete interruption of the transmitted signal).

In order to be conservative, we consider long bursts of packet losses that occur with a probability of $10^{-5}$. Denoting by PER the probability of packet loss taking into account the channel model and packet collisions and $T$ the time sampling interval for vehicle information, the burst size $\Delta$ (in seconds) can be calculated as

$$
\Delta=-5 /\left(\log _{10} \mathrm{PER}\right) T
$$

In our simulations, we considered the sampling rate of $100 \mathrm{~ms}$ as advocated by the ETSI EN 302 637-2 standard.

3) Simulation tool: We used the MATLAB/Simulink environment to model the vehicle dynamics and to implement the control law. We also adopted the WLAN Toolbox of MATLAB to implement the channel configuration for an IEEE 802.11p transmission in order to obtain the Packet Error Rate (PER) taking into account the V2V fading channel aspects, the additive white Gaussian noise, the packet collisions, and other communication parameters as in Table I. The mobility behavior of vehicles is also observed in the MATLAB/Simulink environment as we consider a traffic scenario as in Fig. 1. Both fully cooperative and semi-autonomous control strategies need relative position and longitudinal velocity of the preceding vehicle so we assumed that the measurements are exact, sampled each $60 \mathrm{~ms}$ with $1 \mathrm{~ms}$ delay and done by a long-range radar as in [5]. All the vehicles in the platoon broadcast a 500 
TABLE II

MINIMUM FEASIBLE INTER-VEHICULAR DISTANCE WITH DIFFERENT CONTROL AND COMMUNICATION SCENARIOS

\begin{tabular}{cc||cc}
\multicolumn{2}{c}{ Semi. Auto. with VLC/V2V } & \multicolumn{2}{c}{ PCACC with or without relaying } \\
\hline \hline PER $_{\mathrm{CV}}$ & $\begin{array}{c}\text { Distance } \\
\text { (meter) }\end{array}$ & Mode & $\begin{array}{c}\text { Distance } \\
\text { (meter) }\end{array}$ \\
\hline \hline 0.01 & 1.62 & V2V only & 3.60 \\
0.1 & 2.11 & 5 RSU/Km & 1.38 \\
0.2 & 2.16 & 2 RSU/Km & 1.97 \\
0.5 & 6.55 & $1 \mathrm{RSU} / \mathrm{Km}$ & 9.49 \\
\hline
\end{tabular}

bytes Cooperative Awareness Message (CAM) each $100 \mathrm{~ms}$ on a $10 \mathrm{MHz}$ channel bandwidth [15].

The WLAN Toolbox is used to simulate the wireless environment for $\mathrm{V} 2 \mathrm{~V}$ transmissions. We adopted two different radio link models. The first one is between two consecutive vehicles and its packet error rate will be referred to as $\mathrm{PER}_{\mathrm{CV}}$; it uses a highway line-of-sight (LOS) profile as vehicles in the platoon are close enough. The second link models the communication between the leader and the examined vehicle with a highway non-line-of-sight (NLOS) profile since the leader is less likely to have line of sight with the considered vehicle in the platoon. Furthermore, we perform Monte-Carlo simulations to obtain the SINR (Signal to Interference and Noise Ratio) for each of the vehicles in the platoon considering Winner II Path Loss Model (B1 LOS scenario in [16]) and the interference from other vehicles. We aim to obtain conservative link reliability estimates as in [5].

\section{B. Performance Evaluation}

The focus here is to apply a longitudinal control in the platoon through different communication techniques and analyze the system stability by means of vehicle collisions in some robust and worst-case scenarios. We consider the zeroorder hold mechanism as the holding strategy for the control signal during the periods of packet losses. Furthermore, in all simulations, we focus on obtaining the minimum feasible inter-vehicular distance in the platoon with a collision rate no more than $0.001 \%$ even though we implemented a safety gap distance of $0.5 \mathrm{~m}$ for the emergency braking actuation to avoid collisions in practical settings.

1) Semi-Autonomous Scenario: In this first case, we adopt the semi-autonomous controller fed by VLC or V2V communication links since both of them enable a one-vehicle look-ahead communication topology. The focus is to analyze the impact of the PER in the system dynamics in terms of the minimum feasible inter-vehicular distance. In the V2V approach, the PER is related to the packet collisions and dropouts due to a poor communication link. On the other hand, in the VLC method, it can be interpreted as the measurement errors due to a poor incident angle of the light or due to rainy or foggy environments. In this scenario, we have considered a burst of losses between the leader and the preceding vehicle because it allows us to evaluate the amplification of the error along the tail of the platoon.

The column entitled semi-autonomous VLC/V2V on Table II, provides an insight into the performance in terms of the minimum feasible distance of the platoon taking into account

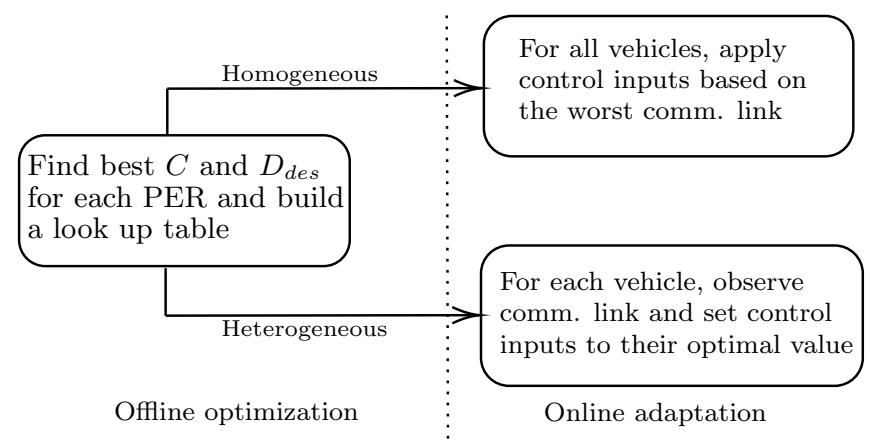

Fig. 3. Offline and online parameter election diagram.

the probability of error rate of the packet for ten vehicles with semi-autonomous control. The results indicate that the packet collisions and dropouts due to a poor communication link or incident angle of the light have a substantial impact on the system performance as a higher PER $_{\mathrm{CV}}$ (PER between two consecutive vehicles) calls for higher inter-vehicular distances to avoid collisions. Note that we do not differentiate here explicitly between V2V and VLC technologies as both may have degraded qualities.

2) Fully Cooperative platoons: We now investigate the performance of fully cooperative platoons, when the platoon communicates with the leader using V2V or RSU relaying, in addition to the neighboring vehicle's communication (using V2V or VLC). Here, we also include a burst loss between the leader and the last two vehicles, due to a congested channel and path loss. This particular burst, defined in (3), was calculated based on the packet error rate of the link between the leader and the last vehicle defined as $\mathrm{PER}_{\mathrm{LLV}}$. We aim to keep a homogeneous control strategy and small-time delay to carry the theoretical results of string stability of [13].

The minimum feasible inter-vehicular distance for PCACC is also presented in Table II. Both pure V2V and RSU relaying modes are presented. When $\mathrm{V} 2 \mathrm{~V}$ is used, there is a need to keep a distance of 3.6 meters between vehicles. This distance reduces to 1.38 meters if RSU relaying is used with a high density infrastructure. This is due to the fact that the RSU selects and manages the radio resources centrally and that it is installed sufficiently high for ensuring a LOS link with vehicles on the highway. However, the advantage of RSU relaying reduces when the infrastructure becomes less dense, and direct $\mathrm{V} 2 \mathrm{~V}$ becomes better for a small RSU density.

\section{Proposed Controller}

This section aims to introduce the proposed dynamic control scheme and to perform a case comparison over static control strategies.

\section{A. Optimal parameters for PCACC}

In contrast to existing works that assume a fixed control strategy or consider only a lower bound parameter as in [8], we propose, as depicted in diagram scheme in Fig. 3, an offline optimization of the control parameters: the weight of the leader's information $(C)$ and the desired distance 
TABLE III

OPTIMUM CONTROL PARAMETERS FOR DIFFERENT PER

\begin{tabular}{c|ccccc}
\hline \hline PER $_{\text {LLV }}$ & 0.05 & 0.2 & 0.3 & 0.4 & $\geq 0.5$ \\
\hline Weight of leader & 0.3 & 0.2 & 0.2 & 0.2 & 0 \\
Desired dist. $(\mathrm{m})$ & 0.37 & 0.47 & 0.58 & 0.58 & 1.08 \\
\hline \hline
\end{tabular}

$\left(D_{d e s}\right)$, for different communication link qualities. Among the control inputs, extensive simulations demonstrate that this pair of parameters has the most substantial impact on the performance of the system. As mentioned before, the first parameter determines the weight of influence of the leader's message in the control algorithm, while the second impacts the inter-vehicular distance for the platoon. However, due to actuator lag and delay in the process, the desired distance does not correspond to the actual average inter-vehicular distance. For any given value of PER (which is the result of the traffic density and the resulting interference) we vary the parameter weight of the leader's information $(C)$ in a range of $0 \leq C<1$ while minimizing the desired distance $\left(D_{\text {des }}\right)$, and we register the average inter-vehicular distance $\left(D_{\text {avg }}\right)$. Then, for the control input pair $\left(C, D_{d e s}\right)$, we build an optimum lookup table as Table III that will serve as reference for the next step. Therefore, from Table III, we see that the best weight of the leader is $C=0.3$ when the channel has a good quality, and is $C=0.2$ for medium channel qualities. For higher error rates, the best choice is zero which corresponds to the case of semi-autonomous control. Notice that in this stage the entire platoon is subject to the same packet error rate based on the last vehicle packet error rate $\left(\mathrm{PER}_{\mathrm{LLV}}\right)$. Furthermore, due to actuator lag and delay the string stability is not guaranteed for all platoon sizes other than those evaluated.

\section{B. Online adaptation of the control parameters}

We now move to the online adaptation of the control parameters based on the observed communication link. To summarize, we perform an offline optimization of the control parameters, above mentioned, followed by an online adaptation based on the communication link quality observed and Table III. We present two implementations of this controller: a centralized homogeneous control where the whole platoon adapts to the worst communication link, and a distributed controller where each vehicle adapts its own control parameters based on each communication link.

1) Homogeneous control: In this case, the whole platoon shares the same information that is transmitted by the leader vehicle to all members. We apply a local controller based on the packet loss observed on the communication link by the leader and the last vehicle $\left(\mathrm{PER}_{\mathrm{LLV}}\right)$. The whole platoon adapts the control inputs corresponding to optimal values computed in the offline optimization for this PER. Thus, the whole platoon is subject to the same adaptive control procedure and we set both control parameters, weight of the leader's message and desired distance, according to its optimal values considering the worst communication link in the platoon.
TABLE IV

CASE COMPARISON FOR THE ONLINE IMPLEMENTATION.

\begin{tabular}{c|c|cccc}
\hline \hline & Parameters & Case 1 & Case 2 & Case 3 & Case 4 \\
\hline \hline \multirow{2}{*}{ Controller } & Weight of the leader & 0.2 & 0 & Dynamic & Dynamic \\
& Desired dist. $(\mathrm{m})$ & 0.58 & 1.03 & Dynamic & Dynamic \\
\hline \hline \multirow{3}{*}{ Outputs } & Average dist. (m) & 1.21 & 1.67 & 1.38 & 1.26 \\
& Minimum dist. (m) & 0.25 & 0.62 & 0.52 & 0.51 \\
& Collisions & 8 & 0 & 0 & 0 \\
\hline \hline
\end{tabular}

2) Heterogeneous Control: An alternate approach corresponds to apply a local controller based on the packet loss observed on the communication link for each vehicle in the platoon. Therefore, based on the offline optimization mentioned before, for each vehicle, we set both control parameters according to their optimal values considering independent communication links. This implies that no additional communication resource is required for the whole platoon to exchanges the exact same information about the control law or communication conditions.

\section{Simulation and case comparison}

A case comparison of different implementations is performed in order to evaluate the proposed scheme over other approaches. We study the occurrence of collisions and compare the inter-vehicular distance of the platoon in a long simulation of 25 minutes in four different cases defined as follows:

- Case 1 - Static PCACC with weight of leader corresponding to $C=0.2$. We apply the desired distance corresponding to the PER between the leader and the middle car in the platoon obtained under average traffic conditions.

- Case 2 - Semi-autonomous (weight of leader set to zero, i.e., $C=0$ ).

- Case 3 - Homogeneous adaptive approach. We apply a uniform control in all the vehicles based on the PER between the leader and the end of the platoon $\left(\mathrm{PER}_{\mathrm{LLV}}\right)$.

- Case 4 - Heterogeneous adaptive approach. We apply a distributed controller that is based on the observed PER for each vehicle.

In all cases, we consider the jammer profile as the pattern in Fig. 2, but repeated 50 times. Another important factor is the traffic density that generates interference and changes the PER for each vehicle. The traffic density varies in the range $\{0,20\}$ vehicle $/ \mathrm{km}$, with the packet error rate between leader to first vehicle set in the interval $\{0.1,0.2\}$ and leader to last vehicle set in the interval $\{0.2,0.6\}$ as shown in Fig. 4. Similarly, the traffic density is generated for the other vehicles in the platoon. Every 2 minutes (4 cycles), the predefined traffic density increases up to its maximum and then decreases to its minimum, and 25 minutes of traffic are simulated overall. We also apply radio error bursts as defined in (3) every $6 \mathrm{~min}$ of simulation.

From Table IV, we notice that Case 1 exhibited 8 collisions while cases 2, 3 and 4 had none. In the first case, the system is assumed to operate in an average traffic condition. However, it can be seen that this is not a safe approach since it does not guarantee a secure operation. In case 2 , despite no collisions, 


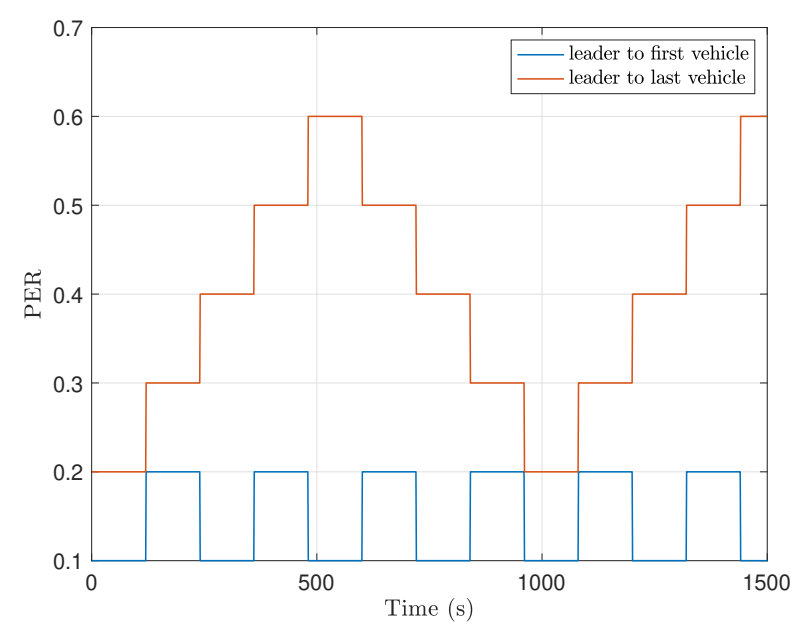

Fig. 4. Traffic density pattern adopted for the worst and the best communication link with the leader.

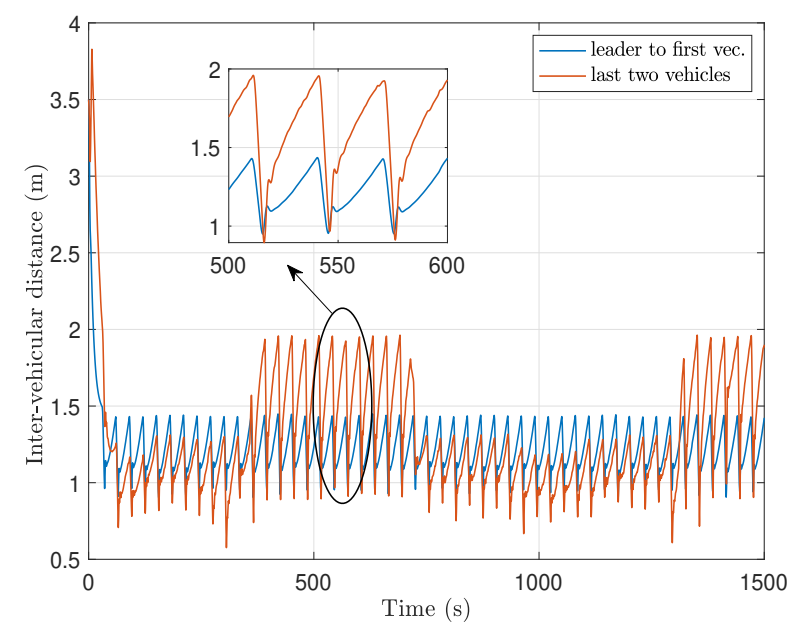

Fig. 5. Illustration of the average inter-vehicular distance for the first two and last two vehicles considering the Case 4 simulation.

there is an increase of $21 \%$ and $33 \%$ in the inter-vehicular distance when compared to cases 3 and 4, respectively. Case 4 is capable to improve approximately $10 \%$ in the inter-vehicular distance when compared to Case 3. Furthermore, it demands fewer communication resources since it adopts a distributed control approach while Case 3 uses homogeneous control that requires an exchange of the same information in the whole platoon.

To give more insights about the platoon dynamics, Fig. 5 illustrates the average inter-vehicular distance for the heterogeneous approach. It can be noticed that vehicles at the beginning are closer while vehicles at the end tend to keep larger distances due to poor communication conditions with the leader. Therefore, the proposed heterogeneous approach is demonstrated to be the best option so that platoon formation remains robust while reducing the average inter-vehicular distance.

\section{CONCLUDING REMARKS}

This paper studies the impact of communication technologies on platooning performance and proposes novel dynamic platoon control algorithms. As of the impact of communication technologies, our results show that relaying of leader information through RSU achieves the best performance, provided that the network is carefully dimensioned for ensuring a low PER. We then proposed a dynamic controller where the quality of the communication link is continuously monitored and the control parameters updated accordingly based on the results of an offline optimization. Our simulation results show that, if the control parameters are not adapted to the channel quality, the semi-autonomous control performs best under bursts of packet losses. However, with the proposed adaptive heterogeneous control approach, using leader information results in a better performance. Our future work will target a more realistic radio link and system models in the presence of V2V relaying.

\section{REFERENCES}

[1] D. Jia, et al., "A survey on platoon-based vehicular cyber-physical systems," IEEE Communications Surveys \& Tutorials, vol. 18, no. 1, 2015, pp. 263-284.

[2] S. Ucar, S. C. Ergen, and O. Ozkasap, "IEEE 802.11p and visible light hybrid communication based secure autonomous platoon," IEEE Transactions on Vehicular Technology, vol. 67, no. 9, 2018, pp. 86678681.

[3] M. Y. Abualhoul, et al., "Platooning control using visible light communications: a feasibility study," IEEE Conf. Intelligent Transportation Systems (ITSC), 2013, pp. 1535-1540.

[4] A. Vinel, L. Lan, and N. Lyamin, "Vehicle-to-vehicle communication in C-ACC/platooning scenarios," IEEE Communications Magazine, vol. 53, no. 8, 2015, pp. 192-197.

[5] V. Vukadinovic, et al., "3GPP C-V2X and IEEE 802.11p for vehicleto-vehicle communications in highway platooning scenarios," Ad Hoc Networks, vol. 74, 2018, pp. 17-29.

[6] J. Ploeg, et al., "Graceful degradation of cooperative adaptive cruise control," IEEE Transactions on Intelligent Transportation Systems, vol. 16, no. 1, 2014, pp. 488-497.

[7] C. Lei, et al., "Evaluation of CACC string stability using SUMO, Simulink, and OMNeT++," EURASIP Journal on Wireless Communications and Networking, 2012., pp. 1-12.

[8] P. Fernandes, and U. Nunes, "Platooning with IVC-enabled autonomous vehicles: strategies to mitigate communication delays, improve safety and traffic flow,", IEEE Transactions on Intelligent Transportation Systems, vol. 13, no. 1, 2012, pp. 91-106.

[9] T. R. Gonçalves, V. S. Varma, and S. E. Elayoubi, "Vehicle platooning schemes considering $\mathrm{V} 2 \mathrm{~V}$ communications: a joint communication/control approach," in Proc. IEEE Wireless Communications and Networking Conf. (WCNC), 2020, pp. 1-6.

[10] R. Rajamani. Vehicle dynamics and control, Springer Science \& Business Media, 2011.

[11] R. Rajamani, et al. "Design and experimental implementation of longitudinal control for a platoon of automated vehicles," J. Dyn. Sys. Meas. Control vol. 122, no. 3, 2000, pp. 470-476.

[12] M. Sybis, et al., "Communication aspects of a modified cooperative adaptive cruise control algorithm," IEEE Transactions on Intelligent Transportation Systems, 2019, pp. 4513-4523.

[13] D. Swaroop, and J. K. Hedrick, "Constant spacing strategies for platooning in automated highway systems," Journal of dynamic systems, measurement, and control, vol. 121, no. 3, 1999, pp. 462-470.

[14] G. Cecchini, et al., "Performance comparison between IEEE 802.11p and LTE-V2V in-coverage and out-of-coverage for cooperative awareness," in Proc. IEEE Vehicular Networking Conf., 2017, pp. 109-114.

[15] P. Alexander, D. Haley, and A. Grant, "Cooperative intelligent transport systems: 5.9-GHz field trials," in Proc. IEEE, vol. 99, no. 7, 2011, pp. 1213-1235.

[16] IST-4-027756, WINNER II, "D1.1.2 V1.2, WINNER II channel models," Tech. Rep. v1.2, 2008. 